\title{
The Relationship between Tensile Strain and Residual Stress of High Strength Dual Phase Steel Sheet
}

\author{
Maoyu ZHAO ${ }^{1, a}$, Zhengzheng MENG ${ }^{1}$, Chunyan TIAN ${ }^{1}$ and Ping LI $^{2}$ \\ ${ }^{1}$ Department of Mechanical Engineering, Hefei University, Hefei 230601, China \\ ${ }^{2}$ School of Materials Science and Engineering, Hefei University of Technology, Hefei 230009, China
}

\begin{abstract}
The relation between residual stress and tensile strain is an important factor for evaluating plastic formation grade of steel sheet. The degree of plastic deformation $(\Delta l)$ and elastic recovery $(\delta)$ were obtained by measuring the length of DP600 steel sheet sample under different tensile test conditions, i.e. five tensile strains $(\varepsilon)$. Furthermore, the average residual stress value in the surface middle (the diameter of $10 \mathrm{~mm}$ ) region of above tensile samples was analyzed by $x$-ray diffraction (XRD) in the crystal plane of (211). By processing the diffraction peak angle $(2 \theta)$ with half width high method (FWHM), the relationship between $\sin ^{2}(\psi)$ and diffraction angle is attained by least squares method. On this basis, a mathematical model was established to correlate the tensile strain with the residual stress in the present study. The results show that the residual stress decreases and the elastic recovery increases with the increase of tensile strain $(\varepsilon \leq 0.205)$. The relation between residual stress and tensile strain can be described with an exponential function . Finally, a function of tensile strain, elastic recovery and surface residual stress is established, by which a reasonable forming condition, viz. $\varepsilon=0.205, \delta=2.65 \mathrm{~mm}$ is determined for achieving the smallest $\sigma_{\psi}$.
\end{abstract}

\section{INTRODUCTION}

The residual stress is a kind of internal deformation coordination of materials, which is caused by constraints or non-uniform deformation, and influences the account of springback. In sheet metal forming represented by stamping and drawing, strain value increase causes the decrease of springback while the increase of the blankholding force and friction between the die and sheet brings about the increase of residual stress ${ }^{[1]}$. So residual stress is an important factor for evaluating the deformation materials ${ }^{[2]}$. The residual stress only takes part of the responsibility of the total measured lattice strain. In fact, the compositional variation inside the material was found to cause greater strain gradient in both ferrite and austenite phases in a dual-phase stainless steel $^{[3]}$.

Residual stress is usually analyzed by a method of $X$ ray diffraction (XRD). Metal material is loaded, uneven elasticity and plastic deformation can emerge. However, owing to the uneven dislocations and slip, a residual stress is generated inside the material in order to balance the non-uniform deformation force after unloading ${ }^{[4]}$. The evaluation of residual stress was performed using the $x$-ray diffraction. It was observed that high pressure allowed the obtaining of high equivalent plastic strain on the top region of the samples, with a subsequent reduction in springback ${ }^{[5]}$. The residual stress in the machined surface has three kinds of distribution: tensile stress, compressive stress and tensile-compressive stress. The maximum tensile stress exists on the outmost surface $^{[6]}$. Detailed X-ray diffraction (XRD) studies of residual stresses of a multilayer or films material are presented $^{[7]}$. The stress-strain curve, proof stress and strain-hardening exponent of the thin film on substrate can be obtained by in-situ XRD stress analysis combined with the tensile test ${ }^{[8]}$. Supported by X-ray diffraction, microstructure analysis and tensile testing, the mechanical response of high entropy alloys to varying strain rates was investigated ${ }^{[9]}$. Tensile tests on commercially pure a-titanium show a three-stage behaviour giving rise to a well on the strain dependence of the work hardening ${ }^{[10]}$.

Therefore, the residual stress affects forming springback that can depict plastic deformation mechanism. Material hardening can reduce the Young's modulus and decrease the amount of springback. By increasing the tensile stress, it increases the material hardening resulting in the reduced amount of springback. Residual stress is an important factor for evaluating the materials deformation, the variation inside the DP steel material can cause greater strain gradient in ferrite phases. The model about strain and residual stress can effectively promote residual stress prediction when DP steel (consists of the martensitic and ferrite phase) was tested on the condition of different strains. 


\section{EXPERIMENTAL}

\subsection{Materials}

The experimental material of this study is dual phase steel of DP600, the microstructure consists of two phases, namely the martensitic (M) strengthening phase on the low strength ferrite (F) matrix with good plasticity. M and $\mathrm{F}$ constitute the plastic properties of DP steel, while the phase grain size and shape are different. The volume fraction of martensitic phase of the DP600 is the 14.2\% and the ferrite phase is $85.8 \%$. The mechanical property parameter of the material is shown in Table $1^{[11]}$. The tensile specimen size is shown in Fig. 1, and experiment result of tension specimen is shown in Fig. 2.

Table 1. Materials property parameters of DP steel

\begin{tabular}{|c|c|}
\hline Elastic modulus, $\mathrm{E}(\mathrm{GPa})$ & 183 \\
\hline Poisson's ration, $v$ & 0.282 \\
\hline Yield stress, $\sigma \mathrm{s}(\mathrm{Mpa})$ & 300 \\
\hline tensile strength $\sigma \mathrm{b}(\mathrm{Mpa})$ & 600 \\
\hline
\end{tabular}

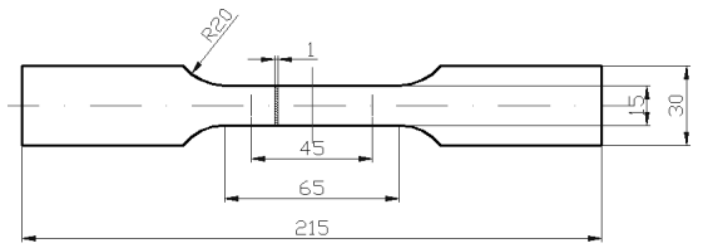

Fig. 1. Dimensions of tension specimen

\subsection{Scheme}

The samples were tested with different tensile strain $(\varepsilon=$ $0.001,0.039,0.100,0.171,0.205)$ at the same stretching rate by means of a uniaxial tensile tester (MTS810).

During the uniaxial tensile experiments of DP600 material, the position of the different tensile strain corresponding to experiment $1-5$ is shown in Fig. 3. Experiment 1 is elastic stage, experiment 2 is yield stage, and experiment $3,4,5$ are plastic deformation stages. In experiment 5 , the strain $\varepsilon$ is 0.205 in the vicinity of the middle of the plastic deformation zone.

Fig. 4 illustrates the uniaxial tensile test specimen, Strain-stress curves of the experiments with the different tensile strain are shown in Fig. 4. The experimental result distribution obtained by calculation as follow, $l$ is the length of the sample after loading, $\Delta l$ is $l-l_{0}$, which the value of $l_{0}$ is the sample original length, equal to 215 $\mathrm{mm}$. The data of the elongation and strain in plastic deformation is displayed in Table 2. Here, $L$ is the maximum movement distance of the specimen before unloading during the tensile test, and the elastic recovery of the experimental samples is expressed as $\delta=L-\Delta l$, as shown in Table 3.
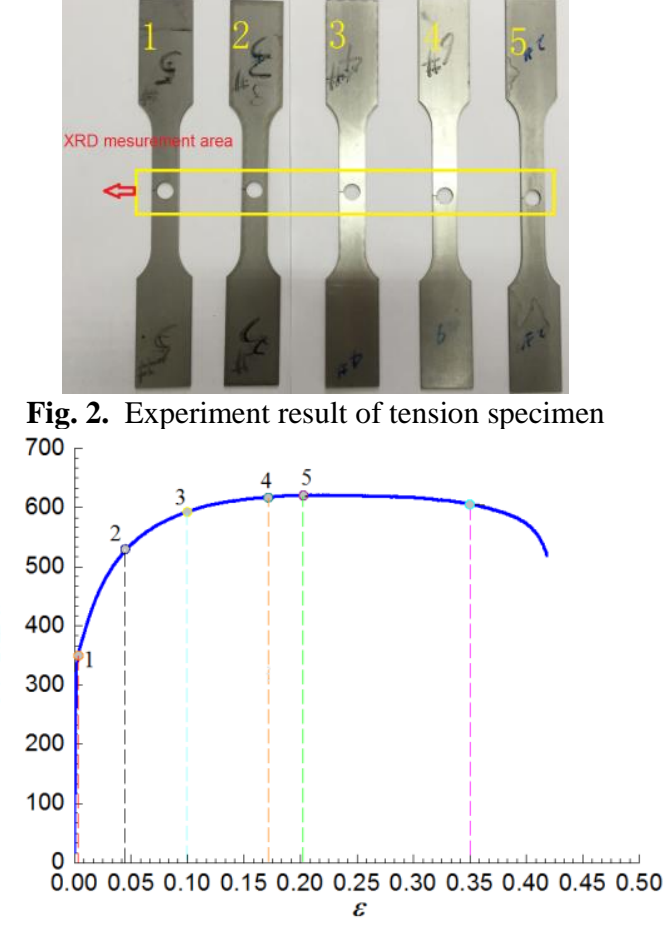

Fig. 3. The tensile stress-strain curve of DP600 material 1-5 point corresponds to the experiment number

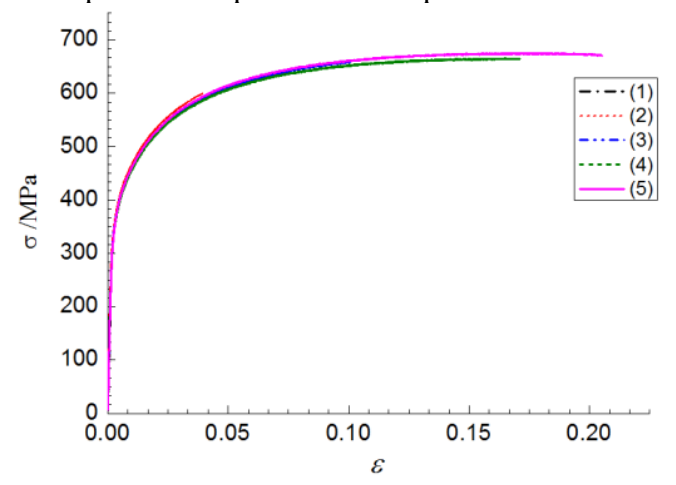

Fig. 4. The tensile stress-strain curve

Table 2. Relation of $\varepsilon$ and $\Delta l$ in the experiment

\begin{tabular}{|c|c|c|c|c|c|}
\hline $\begin{array}{c}\text { Exp. } \\
\text { No. }\end{array}$ & 1 & 2 & 3 & 4 & 5 \\
\hline$\varepsilon$ & 0.001 & 0.039 & 0.100 & 0.171 & 0.205 \\
\hline$l(\mathrm{~mm})$ & 215.5 & 217.6 & 221.5 & 226.0 & 229.6 \\
\hline $\begin{array}{c}\triangle l \\
(\mathrm{~mm})\end{array}$ & 0.5 & 2.6 & 6.5 & 11.0 & 14.6 \\
\hline
\end{tabular}

Table 3. Relation of $\varepsilon$ and $\delta$ in the experiment

\begin{tabular}{|c|c|c|c|c|c|}
\hline $\begin{array}{c}\text { Exp. } \\
\text { No. }\end{array}$ & 1 & 2 & 3 & 4 & 5 \\
\hline$\varepsilon$ & 0.001 & 0.039 & 0.100 & 0.171 & 0.205 \\
\hline$L(\mathrm{~mm})$ & 0.59 & 3.20 & 7.40 & 12.08 & 17.25 \\
\hline$\delta(\mathrm{mm})$ & 0.09 & 0.60 & 0.90 & 1.08 & 2.65 \\
\hline
\end{tabular}

\section{CALCULATION OF RESIDUAL STRESS}

The surface residual stress of the intermediate region was measured by $x$-ray diffraction (XRD). Since the $x$ - 
ray penetration depth is only $10-30 \mu \mathrm{m}$, which the surface residual stress of the specimen can be measured.

\subsection{Computational theory}

The standard $x$-ray diffraction (XRD) method (the $\sin ^{2} \psi$ method) uses the distance between crystallographic planes as a strain gage to determine residual stress value. The relationship between residual strain $\varepsilon_{\psi}$, viz. the relative change of the crystal plane pitch and the change of the diffraction angle $\Delta \theta$ writes

$$
\varepsilon_{\psi}=\frac{d-d_{0}}{d}=\cot \theta \Delta \theta
$$

Where $d, d_{0}$ is diffraction plane spacing with stress and no stress.

If the surface is in a biaxial stress state, when the stress in the sample reference system with z-axis parallel to sample surface normal has only two nonzero components $\sigma_{\mathrm{xx}}=\sigma_{\mathrm{yy}}=\sigma$, and the stress-strain relationship in the material can be described by the Hooke's law with isotropic elastic constants, it can be shown that strain $\varepsilon_{\psi}$ in the direction inclined by an angle $\psi$ from the sample surface normal is given by a simple relation. The strain $\varepsilon_{\psi}$ is

$$
\varepsilon_{\psi}=\frac{1+v}{E} \sin ^{2} \psi \sigma_{\psi}-\frac{2 v}{E} \sigma
$$

In the above equations, $\sigma$ is the stress, $E$ is the Young's modulus, and $v$ is the Poisson's ratio of the material. This is the basis of the well-known $\sin ^{2} \psi$ method $^{[12,13]}$.

The residual stress $\sigma_{\psi}$ can be calculated by rearranging Equations (1) and (2) as

$$
\sigma_{\psi}=\frac{E}{2(1+v)} \cot \theta_{0} \frac{\pi}{180} \frac{\partial\left(2 \theta_{\psi}\right)}{\partial\left(\sin ^{2} \psi\right)}
$$

Assume $K=-\frac{E}{2(1+v)} \cot \theta_{0} \frac{\pi}{180}, \quad M=\frac{\partial\left(2 \theta_{\psi}\right)}{\partial\left(\sin ^{2} \psi\right)}$.

Thus, the $\sigma_{\psi}$ can be written as

$$
\sigma_{\psi}=K \bullet M
$$

Here $\theta_{0}$ is the diffraction angle under the condition of no-stress state.

\subsection{Calculation of the Residual Stress}

In the middle of the tensile specimen material, a circular area with a diameter of $10 \mathrm{~mm}$ was cut out as shown in Fig. 5. It illustrates the test section of residual stress is with a depth of only $10-30 \mu \mathrm{m}$, and the test region is the one with a diameter of $10 \mathrm{~mm}$.

The sample tested region is determined as (211) crystal plane by X'Pert PRO MPD $x$-ray diffractometer. The diffraction peak angle is processed by half width high method (FWHM). The relationship between $\sin ^{2}(\psi)$ and diffraction angle $2 \theta$ is attained by least-squares solution which is demonstrated. The linear slope $K_{l}$ of $\sin ^{2}(\psi)-2 \theta$ curve is shown in Table 4 , and the value $M$ is equal to $K_{l}$.
Table 4. The linear slope $K_{l}$, of experiment specimen

\begin{tabular}{|c|c|c|c|c|c|}
\hline $\begin{array}{c}\text { Exp. } \\
\text { No. }\end{array}$ & 1 & 2 & 3 & 4 & 5 \\
\hline$K_{l}$ & 0.3233 & 0.2696 & 0.2786 & 0.2670 & 0.2583 \\
\hline \multicolumn{5}{c|}{$\begin{array}{c}\text { Residual stress } \\
\text { test section }\end{array}$} \\
\hline
\end{tabular}

Fig. 5. The test section of residual stress

The $E, v, \theta_{0}\left(82.30^{\circ}\right)$ and $M$ value are all substituted into the formula 4 . Finally, the residual stress $\sigma_{\psi}$ of the central region in the sample is shown in Table 5.

Table 5. The residual stress $\sigma_{\psi}$ of experiment specimen (MPa)

\begin{tabular}{|c|c|c|c|c|c|}
\hline Exp. No. & 1 & 2 & 3 & 4 & 5 \\
\hline$\varepsilon$ & 0.001 & 0.039 & 0.100 & 0.171 & 0.205 \\
\hline$\sigma_{\psi}$ & 477.8 & 438.8 & 429.6 & 400.9 & 387.9 \\
\hline
\end{tabular}

\subsection{Results and Discussion}

With the increase of tensile strain, in experiment 1 , the maximum stress $\sigma$ equals $180 \mathrm{MPa}$ when the tensile stress $\varepsilon$ is 0.001 , which is less than the yield strength $\sigma_{\mathrm{s}}$ (300 MPa) of experimental DP600 steel. Therefore, the metal forming of this stage is completely elastic deformation. In experiments $2,3,4,5$, the specimen is stretched corresponding to the strain $\varepsilon$ that equals 0.039 , $0.100,0.171$ and 0.205 , respectively. The material is subjected to plastic deformation in this stage, and the elastic energy stored in the specimen will release and elastic recovery will occur after unloading. While plastic deformation can not be recovered. It can be seen from the calculation that the elastic recovery value $\delta$ are 0.6 , $0.9,1.08$ and $2.65 \mathrm{~mm}$, respectively. Fig. 6 indicates that the elastic recovery value of DP steel material increases with the tensile strain increasing. The plastic deformation of DP steel is more serious, which means the elastic recovery value of the material is larger. Due to the elastic recovery increase when the large plastic deformation of the material turns out after unloading, the large deformation strain of the material also produced finally significant elastic recovery.

The mathematical model of formula 5 can be described by the experimental result of strain $\varepsilon$ and $\sigma_{\psi}$, shown in Table 5. Scrutinizing the experimental $\varepsilon-\sigma_{\psi}$ data, it is found that the exponential-typed mathematical model is capable of describing this $\varepsilon-\sigma_{\psi}$ relation. The sum of squares error is 68.82, coefficient of determination is 0.9442 , root mean squared error is 8.296 . The model is written by

$$
\sigma_{\psi}=469 \times 2.71828^{-0.9342 \varepsilon}
$$

The exponential function reveals that the elastic recovery value increases and the residual stress decreases with the tensile strain $\varepsilon$ increasing, as shown in Fig. 7. The residual stress $\sigma_{\psi}$ is $338 \mathrm{MPa}$ when the strain $\varepsilon$ is 0.35 . With the increase of the strain, the Young's modulus 
decreases from tangent slope of the curve. So the residual stress obviously decreases according to the above formula 5 because the material becomes work hardened during deformation.

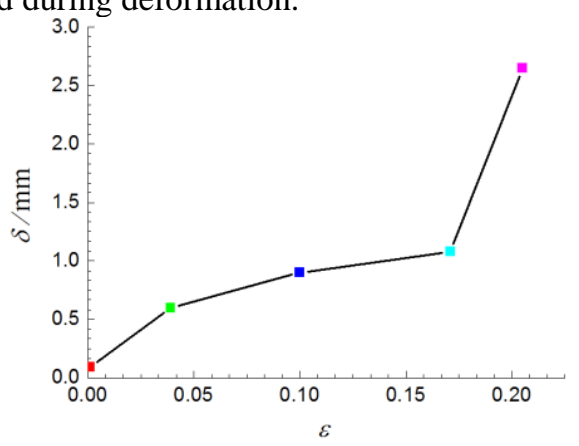

Fig. 6. The curve of strain-elastic recovery value

Controlling springback and residual stress is a guarantee for steel sheet forming quality. When the tensile strain increases, the residual stress and the springback value decreases. The decrease of Young's modulus leads to the reduction of the amount of springback and also the residual stress. So the amount of springback can be reduced by decreasing the residual stress. The smaller the internal balance force corresponding to the residual stress, the more the elastic deformation force is released.

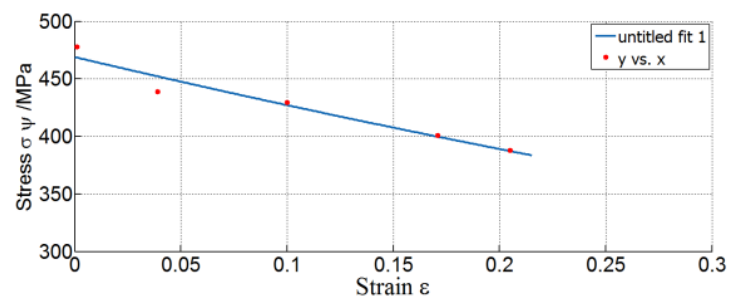

Fig. 7. The residual stress-tensile strain curve

By reducing the residual stress, the amount of springback drops and the forming accuracy can be improved. DP600 material tensile stress-strain curve is shown in Fig. 4. When the tensile strain $\varepsilon$ is 0.35 , it is in the plastic deformation end region. At this time, the residual stress $\sigma_{\psi}$ is $338 \mathrm{MPa}$ with formula 5, which is close to the yield strength $\sigma_{\mathrm{s}}(300 \mathrm{MPa})$ of the DP600 material.

The larger the radius of the die punch is, the smaller the plastic deformation is. So that the residual stress becomes larger, the deformation springback increases. The basis of choice of punch radius may be attained by the relationship between the sheet forming strain and residual stress in the punch area after unloading was probed.

In addition, the results in Fig. 8 only show the relationship between tensile surface residual stress, strain and elastic recovery curve of DP600 material. It can be seen from the fitting surface that experiment $5(\varepsilon, \delta$ and $\sigma_{\psi}$ are $0.205,2.65 \mathrm{~mm}$ and $387.9 \mathrm{MPa}$, respectively) is in a top state, which means that the tensile strength is maximal and the strain may be a more reasonable forming condition because the residual stress is the smallest.

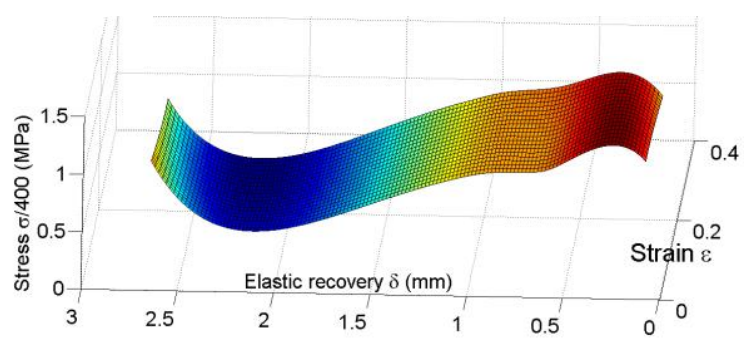

Fig. 8. The tensile residual stress-strain-elastic recover curve of DP600 material

\section{CONCLUSION}

The tensile residual stress of the DP600 steel sheet was studied by uniaxial tensile test under different strain conditions. The relationship between tensile strain and residual stress in the central region of the sample was analyzed. Based on the experimental data, the following conclusions were obtained:

1. The relation between residual stress and tensile strain in the central region of the sample is built to exponent function $\sigma_{\psi}=469 \times 2.71828^{0.9342 \varepsilon}$.

2. Under certain plastic strain conditions $(\varepsilon \leq 0.205)$, elastic recovery value of DP steel increases with the tensile strain increasing, while the residual stress decreases.

3. The tensile strength is maximal when $\varepsilon, \delta$ and $\sigma_{\psi}$ are $0.205,2.65 \mathrm{~mm}$ and $387.9 \mathrm{MPa}$, respectively, which means that the strain may be a more reasonable forming condition because the residual stress is the smallest.

\section{Acknowledgments}

This research was supported by the Anhui Province Natural Science Foundation Project (1308085ME77), Year 2014 Hefei University academic leaders training object project (2014dtr09) and Year 2017 Natural Science Research Project of Colleges and Universities in Anhui Province (KJ2017A540).

\section{References}

1. Asghar. Zajkani, Hamid Hajbarati. An analytical modeling for springback prediction during Ubending process of advanced high-strength steels based on anisotropic nonlinear kinematic hardening model[J]. Adv Manuf Technol. 9, 11 (2016).

2. C. Kahloun, G. Monnet, S. Queyreau, et al. A comparison of collective dislocation motion from single slip quantitative topographic analysis during in-situ AFM room temperature tensile tests on $\mathrm{Cu}$ and Fe crystals [J]. International Journal of Plasticity, 84, 13 (2016).

3. Nan LI, Zhinan AN, and Wenjun LIU, et al. Quanti ${ }^{-}$cation of Compositional and Residual Stress Effects on Lattice Strain in Dual-phase Stainless Steels by Means of Differential Aperture X-ray Micro-diffraction [J]. Acta Metall. Sin. (Engl. Lett.), 26, 6 (2013). 
4. D. Bhattacharyya, W. R. Thorpe and M. J. Painter. Residual stress prediction in large autobody panels[J]. Mater. Shaping technol. 5, 9 (1988).

5. C. Bruni , M. Celeghini and M. Geiger, et al. A study of techniques in the evaluation of springback and residual stress in hydroforming $[\mathrm{J}]$. Int $\mathrm{J}$ Adv Manuf Technol. 33, 11 (2007).

6. Qin Meng-yang, Ye Bang-yan and Jia Xiong, et al. Experimental investigation of residual stress distribution in pre-stress cutting[J]. Adv Manuf. Technol. 65, 7 (2013).

7. M. Sedighi and R. Nazemnezhad. Effect of Peak Positioning Method on Accuracy of X-Ray Diffraction Residual Stress Measurement[J]. Experimental Techniques, 40, 8 (2016).

8. M. Qina, V. Ji, and Y.N. et al. Determination of proof stress and strain-hardening exponent for thin film with biaxial residual stresses by in-situ XRD stress analysis combined with tensile test[J]. Surface \& Coatings Technology, 192, 6 (2005).

9. Wenyi Huo, Hui Zhou and Feng Fang, et al. Strainrate effect upon the tensile behavior of $\mathrm{CoCrFeNi}$ high-entropy alloys [J]. Materials Science and Engineering, A689, 4 (2017).

10. K. E. K. Amouzou, T. Richeton and A. Roth, et al. Micromechanical modeling of hardening mechanisms in commercially pure $\alpha$-titanium in tensile condition $[\mathrm{J}]$. International Journal of Plasticity, 80, 19 (2016).

11. ZHAO Maoyu,CHEN Qianwang, TIAN Chunyan. Spring back predict model of high strength dualphase steel sheet forming $[\mathrm{J}]$. Hot working technology of China Journal. 5, 4 (2014).

12. I.C. Noyan and J.B. Cohen: Residual Stress (Springer Verlag, New York, 1987).

13. H.P. Klug and L.E. Alexander: X-ray Diffraction Procedures, John Wiley \& Sons (New York, NY, 1974). 